\title{
珠穆朗玛峰地区土壤和植被中多环芳烃的含量 及海拔梯度分布
}

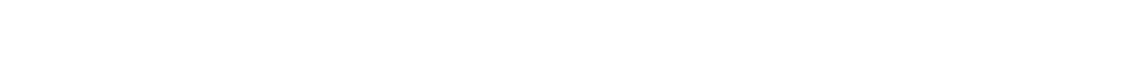

(1) 中国科学院青藏高原研究所, 北京 100085; (2) 山西大学化学化工学院, 太原 030006. E-mail: wangxp@itpcas.ac.cn)

\begin{abstract}
摘要 近年来, 研究者广泛认为高海拔山区为持久性有机污染物的冷凝器和接收器. 虽然高山地区持 久性有机污染物的研究日益增多, 但是对于喜马拉雅山脉地区的研究尚少. 主要研究了珠穆朗玛峰地 区土壤和植被中多环芳烃的含量及其海拔梯度分布模式. 研究结果表明, 珠穆朗玛峰地区土壤中的多 环芳烃属于地球边远地区的水平. 高海拔地区更易于积累挥发性较强的多环芳烃, 易于受到大气远距 离传输污染物的影响. 根据该地区多环芳烃的组成特点, 推断家庭燃烧和汽车尾气的排放是该地区多 环芳烃的主要来源. 季风是将印度等人类活动频繁地区排放的多环芳烃带到珠穆朗玛峰地区的主要贡 献者.
\end{abstract}

\section{关键词 珠穆朗玛峰地区 持久性有机污染物 多环芳烃 海拔梯度分布}

持久性有机污染物(POPs)具有半挥发性, 能够 从水体或土壤中以水蒸汽形式进入大气环境或被大 气颗粒物吸附, 通过大气环流远距离迁移. 在较冷的 地方或者受到海拔高度影响时POPs会重新沉降到地 球上. 而后在温度升高时, 它们会再次挥发进入大气, 进行迁移. 这种过程可以不断发生, 使得POPs物质 可沉积到地球上偏远的极地地区, 导致全球范围的 污染传播. 这也就是所谓的“全球蒸馏效应”或“蚱蜢 跳效应”[1 3] . “蚱蜢跳效应”成功地解释了有机污染物 通过蒸发、大气传输进而迁移到极地地区的机制 ${ }^{[4]}$. 鉴于POPs物质在区域乃至全球尺度下的大气输送过 程可能对高纬度地区生态环境造成严重的影响, 已 经有较多的工作集中研究极地(主要是北极)环境中 POPs物质的分布、迁移及其组成变化的规律 ${ }^{[5 \sim 10]}$. 目 前欧美一些学者也已经在阿尔卑斯山和北美洛基山 脉开展了大气 ${ }^{[11]} 、$ 湖泊 $^{[12,13]} 、$ 冰 ${ }^{[14]}$ 中POPs物质的 研 究.

近年来, 如何定量研究高山地区的污染程度日 益引起人们的注意 ${ }^{[4]}$. 研究高山地区中的有机污染物 对人类、水体和陆地生态系统的潜在影响具有很重要 的意义 ${ }^{[15]}$. 高山地区的有机污染物除了会对高山地 区自身的生态系统造成威胁之外, 高山地区冰雪和 湖泊中的有机污染物还会对人类的饮用水资源和农 田供给水资源造成潜在的影响. 此外, 高海拔处的植 被也有可能受到有机污染物的危害, 有机污染物可 以被富集、吸收并储存在高寒植物体内, 而山区家畜
和高等动物则可能通过食用植被的方式在其体内积 累污染物 ${ }^{[16]}$. 因此, 研究高海拔地区植被和土壤中有 机污染物的水平对于评价该类污染物可能对人类和 动物健康的危害有很重要的意义.

虽然很多作者开始关注高山地区持久性有机污 染物的分配和传输, 然而对于喜马拉雅山地区污染 物的报道却很少. 喜马拉雅山位于印度和中国之间, 珠穆朗玛峰(海拔 $8844.3 \mathrm{~m}$ ), 是世界最高峰. 珠穆朗 玛峰地区与人口密集的国家相毗邻，如印度、巴基斯 坦、孟加拉国和中国. 在过去的几十年中, 印度次大 陆和中国曾大量使用过有机氯农药, 如六六六 $(\mathrm{HCH})$ 和滴滴涕 $(\mathrm{DDT})^{[17]}$. 而且随着人口的急剧膨胀, 人类 使用煤和石油的燃烧活动、汽车尾气排放、垃圾焚烧 或森林大火等人为事件增加, 使得印度和中国每年 都有大量的多环芳烃(PAHs)排放到地表和空气当中. 在环境中部分多环芳烃已被证实对人体具有致癌与 致突变性. 这些有机污染物一旦进入几千米高的主 要空气环流, 就很容易被长距离运送, 从而进入珠穆 朗玛峰地区. PAHs大多吸附在大气和水中的微小颗 粒物上, 大气中的PAHs 可以通过干沉降和湿沉降(大 气降水)的方式降落到地表, 从而污染土壤和地表水 源. 研究表明, 除了工业排污外, 大气降水是地表水 中多环芳烃的主要来源 $[18]$. 珠穆朗玛峰地区较低的 气温、较高的海拔以及强烈的降雪过程将加速有机污 染物在高原上的凝结和聚集. 由于PAHs在水中溶解 度较低而其亲脂性又较强，因此该类化合物易于从 
水中分配到沉积物、有机质、土壤及生物体内, 其结 果使土壤及生物体中多环芳烃的残留浓度较高 [19] 本文通过测定珠穆朗玛峰地区不同海拔梯度处土壤 和植被中多环芳烃的含量研究了多环芳烃的含量随 海拔梯度的变化趋势, 并利用多环芳烃的组成比率 讨论了珠穆朗玛峰地区多环芳烃的主要来源, 最后 结合该地区季节性气候特点探讨了多环芳烃的传输 途径.

\section{1 研究区概况}

研究地点位于定日县曲宗乡一一珠穆朗玛峰线 布河谷地区, 海拔梯度为 4600 5620 m (图 $1^{[20]}$ ). 研 究区的植被类型为棘豆 $(O$. glacialis Benth. ex Bge)和柔 籽草(T. rupifragum Schrenk), 土壤类型为寒冻钲土 ${ }^{[20]}$. 研究地区位于珠穆朗玛峰核心保护区内, 其气候特点 与珠穆朗玛峰地区的气候一致, 冬季受季节性南下的 西风带控制, 夏季受印度洋北上的夏季风影响.

\section{2 样品采集与萃取}

2005 年 4 月, 在线布河谷 $\left(28.30^{\circ} \mathrm{N}, 87.04^{\circ} \mathrm{E} \sim\right.$ $\left.28.29^{\circ} \mathrm{N}, 87.03^{\circ} \mathrm{E}\right)$ 沿不同的海拔梯度采集柔籽草、棘 豆和土壤样品(详见表 1). 植物及土壤样品用铝箔包 好, 放入棕色样品瓶中冷冻保存. 在进行索氏提取之 前, 所有的样品都保持冷冻状态. 将所得样品冷冻干 燥后, 分别称取土壤 (10 g), 植物样品 (5 g) 置于索氏 提取器内, 加入菲-D10 和菲-D12 作为回收率指示物,
以混和溶剂正己烷: 丙酮 $=1: 1$ 进行抽提 $24 \mathrm{~h}$, 共约 30 个循环, 收集萃取液. 将萃取液浓缩后用硅胶柱净化. 用二氯甲烷: 正己烷 $=2: 1$ 的溶剂洗脱层析柱进而获 得芳烃组分. 用旋转蒸发仪将该组分浓缩, 氮气吹至 $1 \mathrm{~mL}$, 加入内标萠-D10, 并转入进样瓶中待测. 此外, 称取 $0.1 \mathrm{~g}$ 干燥后的土壤在日本岛津总有机碳分析仪 上对土壤中的总有机碳进行测定.

表 1 不同海拔高度所得样本数及土壤的总有机碳含量 (TOC)

\begin{tabular}{ccccc}
\hline 海拔 $/ \mathrm{m}$ & 柔籽草 & 棘豆 & 土壤 & $\begin{array}{c}\text { 土壤 } \mathrm{TOC} \\
/ \mathrm{mgC} \cdot \mathrm{g}^{-1}\end{array}$ \\
\hline 4600 & 3 & 2 & 5 & 10.4 \\
4800 & 3 & 2 & 5 & 2.86 \\
5210 & 2 & 2 & 4 & 2.87 \\
5440 & 3 & 3 & 6 & 2.94 \\
5620 & 2 & 2 & 4 & 2.88 \\
\hline
\end{tabular}

\section{3 样品分析及质量保证}

测定样品中多环芳烃(美国 EPA 所优先控制的 16 种多环芳烃)所用仪器为离子阱 GC-MS (Finnigan Trace GC/PolarisQ), 配有 Triplus 自动进样器; $30 \mathrm{~m} \times$ $250 \mu \mathrm{m}, \mathrm{DB}-5 \mathrm{MS}$ 毛细管柱分离, 高纯氦气做载气, 恒定流速为 $1.0 \mathrm{~mL} / \mathrm{min}$, 不分流模式进样 $(1 \mu \mathrm{L})$. 为了 得到更好的测试灵敏度, 本文选择了串连质谱模式 (MSMS)对样品进行了分析. 气相色谱的升温程序为: $50^{\circ} \mathrm{C}$ 停留 $2 \mathrm{~min}$; 以 $20^{\circ} \mathrm{C} / \mathrm{min}$ 的速度升至 $180^{\circ} \mathrm{C}$; 再以

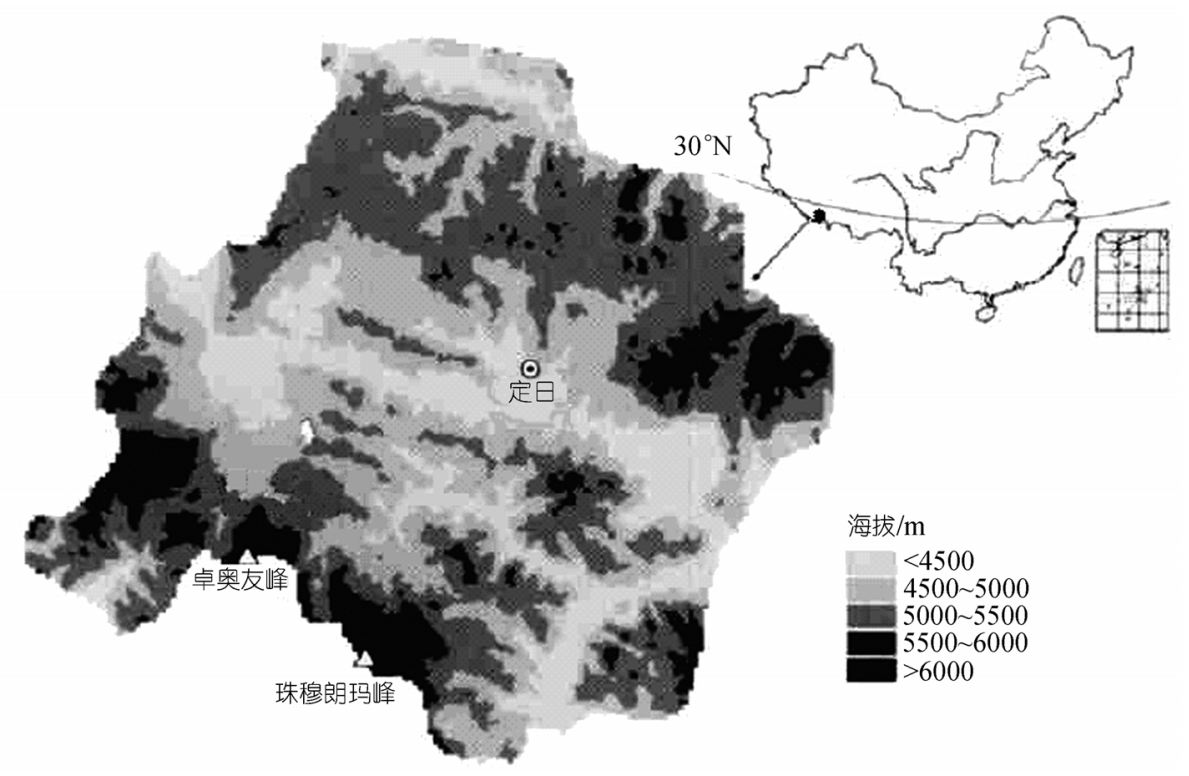

https://engine.sci图1 B.c研究区地理位置图2006-51-21-2517 
$4^{\circ} \mathrm{C} / \mathrm{min}$ 的速度升至 $250^{\circ} \mathrm{C} ; 2^{\circ} \mathrm{C} / \mathrm{min}$ 的速度升至 $280^{\circ} \mathrm{C}$, 停留 $2 \mathrm{~min}$; 最后以 $10^{\circ} \mathrm{C} / \mathrm{min}$ 的速度升至 $300^{\circ} \mathrm{C}$, 停 留 $5 \mathrm{~min}$. 用上述色谱及质谱方法测定回收率指示物 质, 菲-D 10 的回收率在 $85 \% \sim 110 \%$, 菲-D 12 的回收率 在 73\% 89\%之间. 此外, 我们还测定了 NIST 标准参 考物质(NIST 1944 纽约/新泽西排水沟沉积物)中多环 芳烃的含量, 并与其 NIST 参考值进行了比较. 结果 表明对于芳烃类物质本文的测试结果在 $65 \%$ 110\% 的水平上接近于标准值. 对每个分析样品(样品、方法 空白、基质加标、加标空白)都要添加回收率指示物, 以控制整个分析流程的回收率. 每个工作日都用标 准物质校正仪器, 而且每分析 15 个样品要加测方法 空白和加标空白的样品.

\section{4 结果与讨论}

\section{1 土壤及植被样品中多环芳烃的含量}

表 1 列出了所采集样本的样本数及土壤样品的 总有机碳含量. 荡、菲、范是土壤中普遍存在的多环 芳烃(见表 2). 芴的含量约占土壤中总多环芳烃含量 的 $50 \%$, 而菲约占总芳烃的 $20 \%$. 在土壤样品中没有 测到高分子量的芳烃, 诸如二苯并 $[\mathrm{a}, \mathrm{h}]$ 蒽、茚 并 $[1,2,3-\mathrm{cd}]$ 萠、苯并 $[\mathrm{ghi}]$ 菲、苯并 $[\mathrm{a}]$ 葸、苯并 $[\mathrm{k}]$ 苂 蒽和苯并 $[a]$ 芘). 其中苯并 $[b]$ 苂蒽也只在个别样品中 被检测到(见表 2). Jones等人 [21]曾经对英国威尔士地 区表层土壤 $(0 \sim 5 \mathrm{~cm})$ 中的多环芳烃进行了全面的测 定, 结果发现该地区土壤中多环芳烃的含量从 108 $\mathrm{ng} / \mathrm{g}$ 到 $54500 \mathrm{ng} / \mathrm{g}$ 不等. 污染物水平大致可以分为三 个等级, 分别对应于偏远地区土壤(多环芳烃含量约 在几百个 $n g / g)$, 农田土(几千个 $n g / g$ )和城市土壤(上 万个 $n g / g)$. Jones等人 ${ }^{[211}$ 还认为偏远地区因远离人类
活动土壤中的多环芳烃一般小于 $600 \mathrm{ng} / \mathrm{g}$, 而城市土 壤则一般都会大于 $600 \mathrm{ng} / \mathrm{g}$. 从我们的实验数据来看, 珠穆朗玛峰地区土壤中多环芳烃的含量在 168 595 $\mathrm{ng} / \mathrm{g}$ (干重)的范围之内, 基本属于偏远地区土壤的水 平. 此外, Maliszewska-Kordybach也根据土壤中多环 芳烃的含量将波兰农田土进行了分类, 并提出了相 应的环境标准值 ${ }^{[22]}$. 他认为多环芳烃浓度在 200 600 $\mathrm{ng} / \mathrm{g}$ (干重)时, 该土壤基本属于未被污染的水平; 在 600 1000 $\mathrm{ng} / \mathrm{g}$ (干重) 时土壤为轻度污染; 而超过 $1000 \mathrm{ng} / \mathrm{g}$ (干重)时则表明该土壤为重度污染土. 由此 可见，珠穆朗玛峰地区土壤中的多环芳烃应当可以 代表北半球远离污染源地区土壤的环境背景值. 南 极土壤中多环芳烃的研究主要集中对科学考察站附 近的土壤进行研究. 诸如, 南极McMurdo站多环芳烃 萗、范、二氢范和菼葸的含量分别在 27000,17800 , 15700 和 $13300 \mu \mathrm{g} / \mathrm{g}$ 的水平 ${ }^{[23]}$, 远高于本文所得的数 据. 另外一些研究主要针对科学考察站垃圾堆放地 的土壤污染问题 ${ }^{[24]}$. 结果发现石油制品的随意堆放 已经造成南极土壤中PAHs的污染. PAHs的总量在 41 8105 ng/g的范围内, 也高于珠穆朗玛峰土壤中 PAHs的数据. 由于南极地区的工作主要围绕科考站 附近的土壤而展开, 科考站附近受人类活动影响太 大, 其中多环芳烃的污染也多为石油泄漏导致的. 因 此无论从地理位置的选取上还是研究的普遍意义上 来看这些PAHs的数据都不能代表地球偏远地区 $\mathrm{PAHs}$ 的水平. 北极PAHs的研究主要集中与水体、河 流三角洲以及湖泊沉积物等介质. 从沉积物的数据 看, 多环芳烃的总量在 $220 \sim 1200 \mathrm{ng} / \mathrm{g}$ 的水平 ${ }^{[25]}$, 高 于珠穆朗玛峰地区土壤中多环芳烃的含量.

表 2 土壤及植物样品中多环芳烃的含量 $(\mathrm{ng} / \mathrm{g}$, 干重)

\begin{tabular}{|c|c|c|c|}
\hline 目标化合物 & 土壤 & 柔籽草 & 棘豆 \\
\hline 二氢范 & $32.3 \sim 152$ & $0.004 \sim 0.353$ & $0.006 \sim 1.41$ \\
\hline 范 & $23.5 \sim 94.6$ & $0.009 \sim 2.64$ & $0.063 \sim 20.1$ \\
\hline 葸 & $0.903 \sim 4.83$ & $0.391 \sim 2.65$ & $0.372 \sim 2.56$ \\
\hline 苂葱 & $5.87 \sim 24.3$ & $1.01 \sim 7.11$ & $1.66 \sim 8.17$ \\
\hline 芴 & $43.9 \sim 233$ & $1.67 \sim 23.2$ & $1.07 \sim 26.4$ \\
\hline 菲 & $21.8 \sim 102$ & $2.96 \sim 52.8$ & $39.5 \sim 80.6$ \\
\hline 芘 & $0.887 \sim 3.16$ & $0.693 \sim 1.99$ & $0.771 \sim 3.48$ \\
\hline 䓛 & $1.25 \sim 4.04$ & $0.214 \sim 1.76$ & $0.917 \sim 3.06$ \\
\hline 苯并 $[\mathrm{a}]$ 葸 & ND & $0.123 \sim 1.25$ & $0.642 \sim 1.75$ \\
\hline 苯并 $[\mathrm{b}]$ 荧蒽 & $0.461 \sim 2.89$ & $0.506 \sim 3.85$ & ND \\
\hline 苯并 $[\mathrm{k}]$ 苂葱 & ND & $0.308 \sim 1.86$ & ND \\
\hline 苯并 $[\mathrm{a}]$ 芘 & ND & $0.113 \sim 1.91$ & ND \\
\hline 多环芳烃总量 & httos://engii168 595 & /c 12.8 $ه 92.551-21-2517$ & $49.4 \sim 146$ \\
\hline
\end{tabular}


据报道，燃烧排放源中葱与菲的含量相近，而蒽 比菲易于光降解 ${ }^{[26]}$, 因此土壤中菲/葸的值就可以作 为判断多环芳烃是远距离传输而来还是局地排放的 一个参数. 珠穆朗玛峰土壤中菲/葸的平均值为 36 , 这说明珠穆朗玛峰地区的多环芳烃主要为大气远距 离传输而来(葱已大量降解), 局地燃烧的贡献较小. 类似地, 土壤中苂葸和萠的比率也是用于证明多环 芳烃是否经历远源传输的有用指标. 荻葱在热力学 上比萠稳定, 较高的菼葱/萠值往往意味着多环芳烃 是从遥远地区经过大气颗粒物的搬运过程而到达采 样地点 ${ }^{[27]}$. 相反, 石油裂解物中菼葸/萠的值往往小

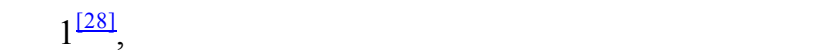
泄漏而导致的多环芳烃污染. 本文的测试结果表明 珠穆朗玛峰地区土壤中菼葱与萠的比值在 6 18 之间 波动, 这说明在该地区石油泄漏不是多环芳烃的主 要来源, 而大气对燃烧物的远距离输送是珠穆朗玛 峰地区土壤中存在多环芳烃的原因.

与土壤不同的是, 我们在柔籽草和棘豆样品中 测到了共 12 种多环芳烃. 植物样品中检测到了苯 并 $[a]$ 葱、苯并 $[k]$ 苂葱和苯并 $[a]$ 萠, 而这些芳烃却没 有在土壤样品中被检测到. 这说明与土壤相比, 富含 脂类的植被更容易吸收并富集大气中的多环芳烃. 在所检测到的 12 种多环芳烃中, 菲依然是含量最高 的组分. 从表 2 可以看出, 植被中低分子量的多环芳 烃(萗、范、二氢范、菲、芴、葱、萠，分子量 $<202$ ) 的含量一般在几十个 $n g / g$, 是含量较高的组分; 而高 分子量的多环芳烃(分子量>202)的含量则小于 $5 \mathrm{ng} / \mathrm{g}$.
Wild等人 ${ }^{[29]}$ 曾经报道了英格兰南部松针样品中各类 多环芳烃的含量, 其中菲、芴和范的含量一般在几百 个 $n g / g$ 的水平, 而其他低分子量多环芳烃的含量在几 十个 $n g / g$, 高分子量的多环芳烃的含量最少, 大约几 个 $n g / g$. 本文所报道的数据与Wild等 ${ }^{[29]}$ 的数据非常 吻合, 这一方面说明植被可以吸附大气传输来的污 染物质, 是反映大气污染的良好介质, 另一方面也说 明珠穆朗玛峰地区大气中多环芳烃的含量应该与英 格兰南部地区相近. 此外, 我们在植被样品中也观察 到了异常高的菲/蒽(15 73)和菼蒽/萠(0.698 4.85)值. 植被中多环芳烃含量的数据也说明珠穆朗玛峰地区 的该类污染物是由珠穆朗玛峰毗邻地区的污染物经 过远距离大气传输而来的.

\section{2 多环芳烃含量随着海拔高度的变化}

我们分别对不同海拔高度样品中多环芳烃的含 量与海拔高度进行了相关分析. 对于土壤样品而言, 范、二氢范、菲、芴、葸和萠与海拔高度呈正相关; 菼 蒽和 的含量并不随海拔升高有明确的变化趋势; 苯并 [b]苂葸的含量随着海拔的升高有降低的趋势, 呈负相关关系(图 2). 一般来说, 高山地区中多环芳 烃的主要来源就是大气沉降. 低分子量的多环芳烃 多以气态的形式存在于大气中, 它们相对易挥发, 易 于在大气中迁移到更遥远的地方, 进而易于在高海 拔的位置积累. 与此同时这些易挥发的多环芳烃遇 到冷空气的时候还可以冷凝并吸附在大气颗粒物上, 然后随着降雪等沉降过程进入地表系统 ${ }^{[30]}$. 然而随 着多环芳烃环数的增加, 分子量的增大, 它们变得更

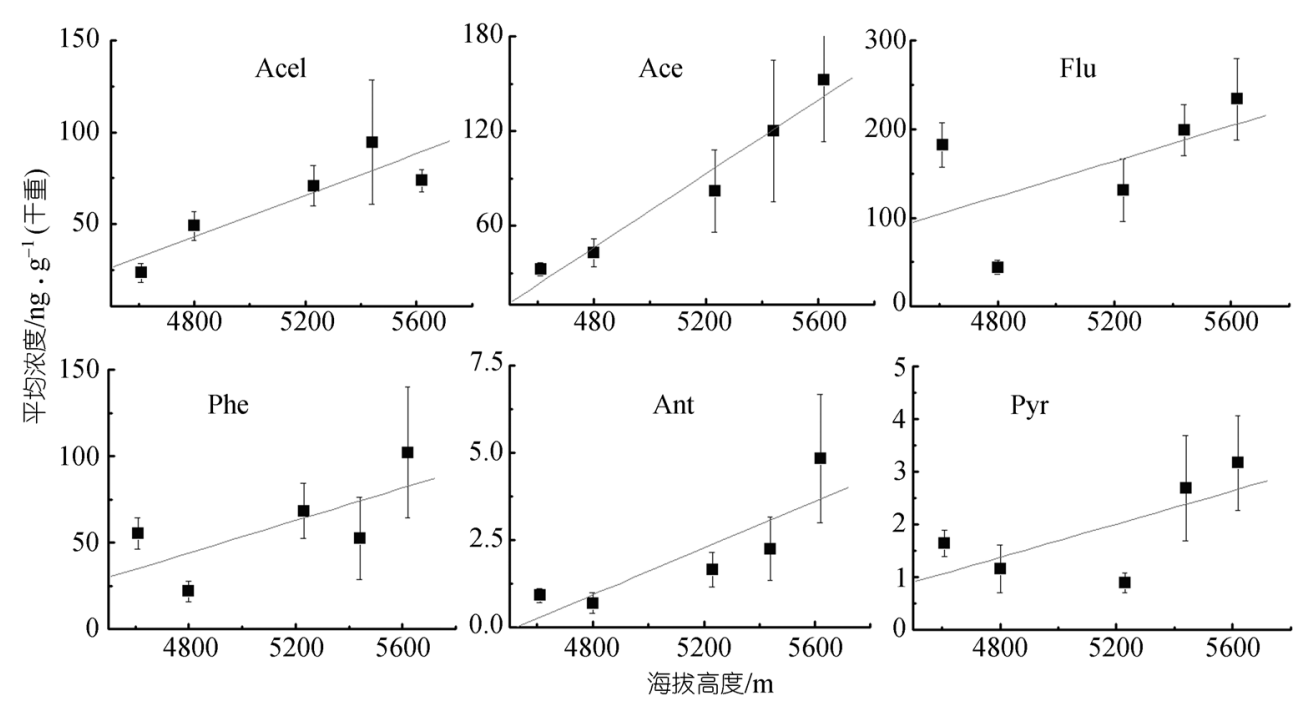

图 2 珠穆朗玛峰地区土壤中多环芳烃的含量随海拔高度的变化趋势 

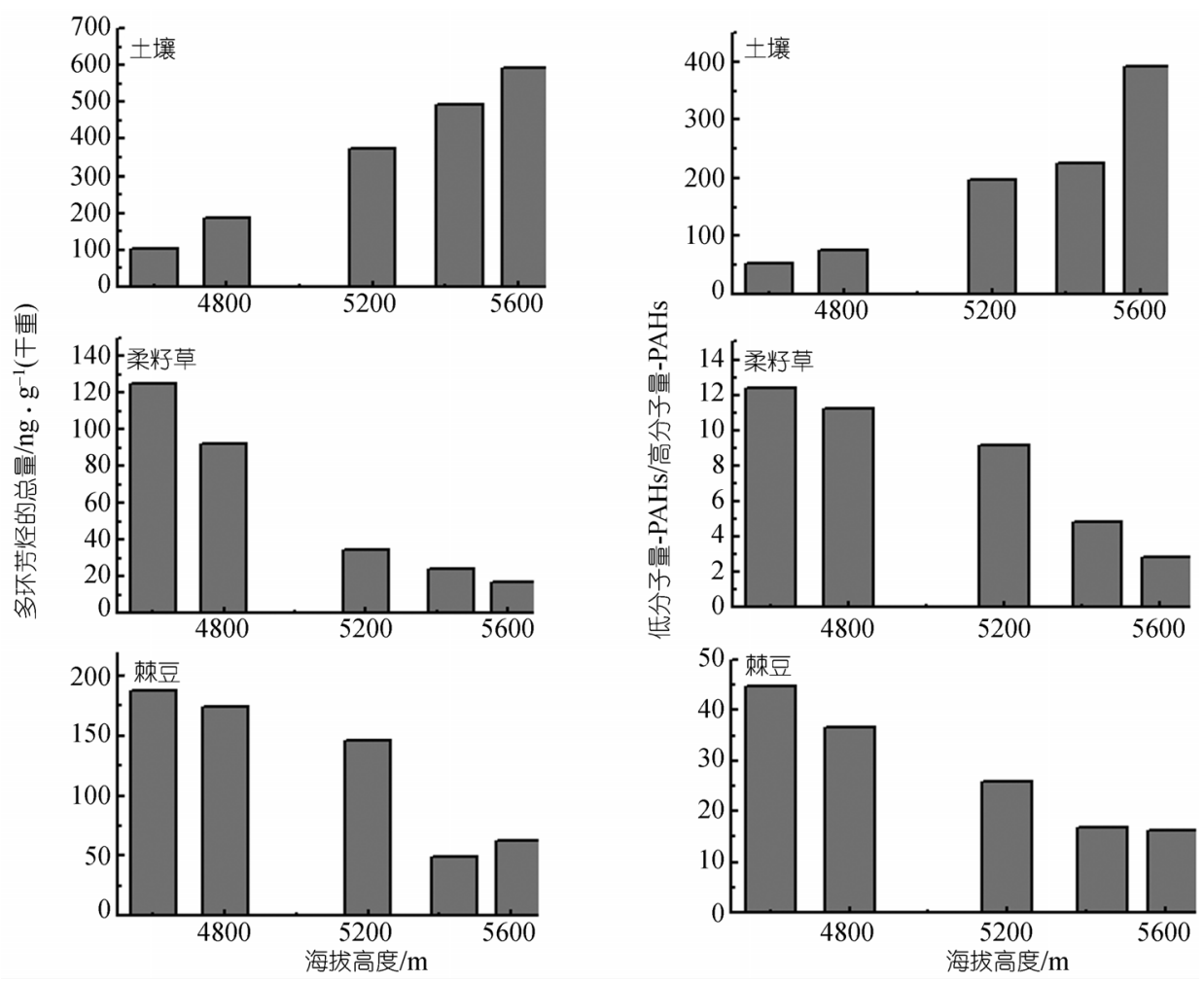

图 3 土壤及植物样品中总多环芳烃以及低分子量 PAHs/高分子量 PAHs 随海拔高度的变化趋势

倾向于吸附在大气颗粒物上进行迁移. 5 或 6 环多环 芳烃, 如苯并 $[\mathrm{b}]$ 苂葸、苯并 $[\mathrm{k}]$ 苂葸、苯并 $[\mathrm{a}]$ 萠、二 苯并 $[\mathrm{a}, \mathrm{h}]$ 蒽、苯并 $[\mathrm{ghi}]$ 萠和狮并 $[1,2,3-\mathrm{cd}]$ 芘, 它们则 主要与大气颗粒物结合 ${ }^{[31]}$. 因此, 这些较重的多环芳 烃不易于长距离传输. 一旦它们随着大气颗粒物沉 降在地表, 就不会再次挥发进行二次迁徙. 低分子量 多环芳烃的含量随着海拔升高而升高, 而高分子量 的多环芳烃则主要集中在海拔较低的位置, 这与多 环芳烃其自身的挥发能力有直接的关系 ${ }^{[31]}$. 从图 3 中 我们可以看出土壤中多环芳烃的总量随海拔高度的 增加也呈上升的趋势, 这是因为低分子量的多环芳 烃占总多环芳烃含量的 $80 \%$ 以上，低分子量多环芳 烃随海拔的变化决定着多环芳烃总量的变化趋势. 土壤样品中菲与葱的比值和苂葱与萠的比值也都随 着海拔的升高而升高. 这一方面可能是由于高海拔 比低海拔处接收到了更多远距离输送而来的污染物 质, 另一方面的原因可能是高海拔处强烈的太阳辐 射也会导致易光降解有机污染物含量的降低.

与土壤不同的是, 我们发现所有在植被样品中 检出的多环芳烃都与海拔高度呈负相关(图 4). 不但 分子量较大的苯并 [a]葱和 与海拔呈明显的负相关
关系，其他低分子量的多环芳烃也呈现负相关关系. 通过植被中的有机污染水平可以间接反映大气污染 的水平. 但是, 由于植被只能吸附大气中以气态存在 的污染物, 而极少吸收由湿沉降捕获到的污染物, 因 此植被中污染物只能反映大气污染物干沉降的水平. 土壤中污染物的含量是大气干湿沉降的总和. 已经 有很多研究发现由植被所揭示的污染物海拔梯度分 配模式与土壤所揭示的不同 ${ }^{[32,33]}$. 究其原因应该是 土壤和植被反映的大气沉降模式不完全相同所造成 的. 珠穆朗玛峰植被中多环芳烃含量随海拔升高而 减少说明该地区高海拔处以气态存在的多环芳烃的 含量较少, 多环芳烃的大气干沉降随海拔的升高而 降低. 从土壤中多环芳烃的含量与海拔的正相关关 系来看, 湿沉降是珠穆朗玛峰地区多环芳烃由大气 沉降到地表的主要方式. 珠穆朗玛峰地区强烈的降 雪过程清除了大气中的多环芳烃.

多环芳烃在大气传输的过程中显然要经历各种 复杂的生物化学过程. 最主要的过程应当包括挥发、 吸附于颗粒物表面、沉降(降雪清除)、光降解、光反 应(臭氧和氮氧化物)和生物降解. 本文中多环芳烃的 总量随海拔高度的变化趋势显然是物质挥发能力、湿 


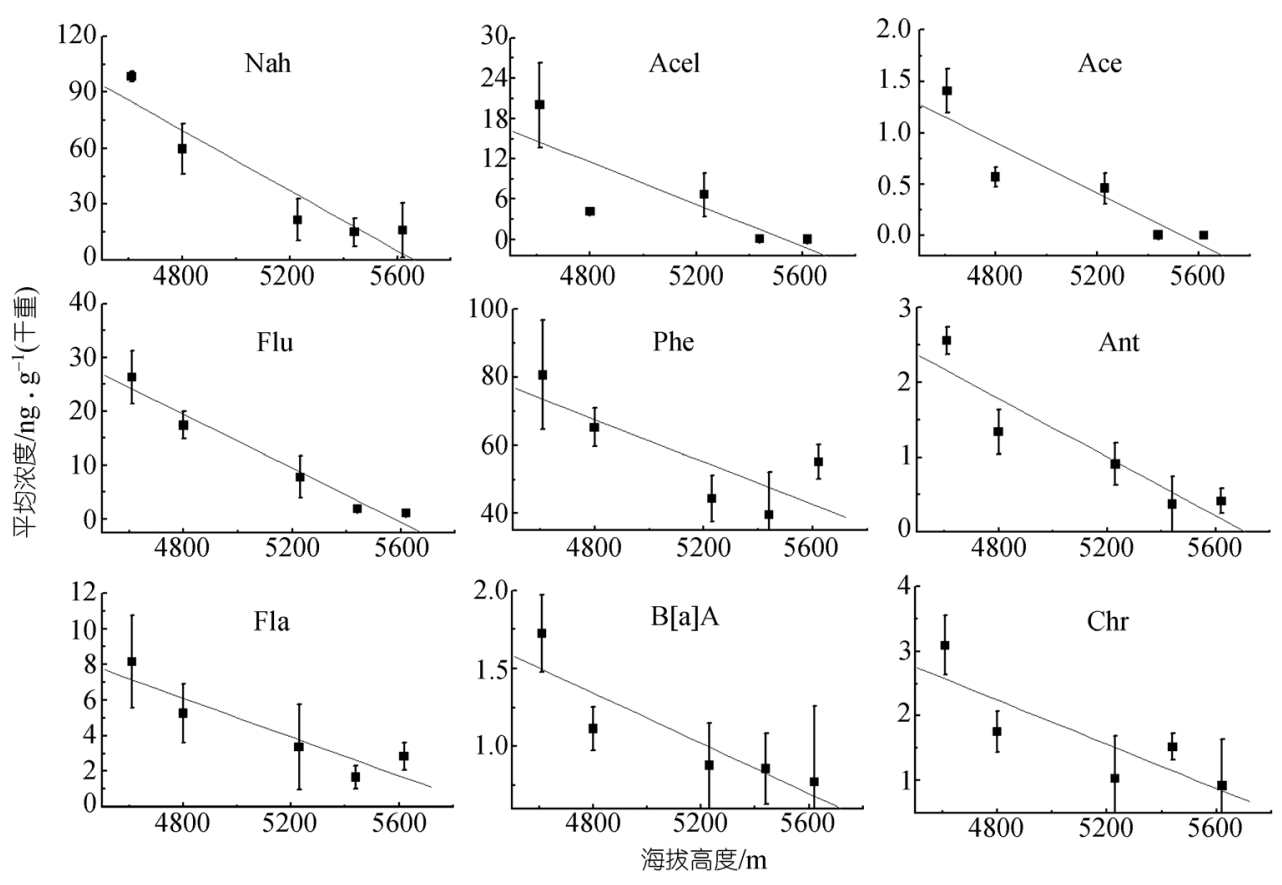

图 4 珠穆朗玛峰地区柔籽草中多环芳烃的含量随海拔高度的变化趋势

沉降的清除能力和被降解能力等因素综合作用的结 果. 易挥发PAHs易于吸附在颗粒物表面进行迁移同 时也易于在高海拔处通过湿沉降而得到清除. 已有 文献报道多环芳烃的生物降解往往与介质的营养程 度有直接的关系, 并不随海拔和温度的变化有明显 的改变 ${ }^{[34]}$, 因此我们暂不考虑生物降解的影响. 因为 高海拔处紫外线强烈, 多环芳烃的光降解程度加剧 进而导致了多环芳烃的含量在高海拔处显著降低. 本文中气态多环芳烃的含量随海拔高度的增加而减 少可能就是光降解程度加剧的结果. 而土壤中多环 芳烃的含量却随海拔的增高而上升, 应该是物质的 挥发能力以及降雪的清除能力起到了主要的作用.

综上所述，珠穆朗玛峰地区石油泄露造成的多 环芳烃的污染基本不存在, 多环芳烃主要来自燃烧 排放. 而且相对于低海拔处, 高海拔处积累了更多的 挥发性多环芳烃. 与此相反, 高环数的多环芳烃则主 要集中在低海拔处. 高海拔强烈的降雪不但捕获了 以气态形式存在的多环芳烃也捕获了吸附于大气颗 粒物中的多环芳烃.

\section{3 人类燃烧活动对珠穆朗玛峰地区多环芳烃的贡献}

家庭或民用燃烧产生的烟气以及石油产品在不 完全燃烧时的排放产物是环境中因人为因素产生多
环芳烃的两大主要来源. 母体芳烃一般是由燃料和 植物残体燃烧以及自然成岩过程产生的. 对于由燃 烧而产生的多环芳烃而言, 低分子量芳烃主要产生 于低温或者较高温度的燃烧过程, 而高环的多环芳 烃则主要由人为的高热燃烧所产生, 更能反映人类 活动的状况. 同时, 石油的燃烧残迹物中也含有较高 丰度的 2, 3 环多环芳烃. 我们用低分子量多环芳烃 (分子量 $<202$ )与高分子量多环芳烃(分子量>202)的比 值(LMW-PAHs/HMW-PAHs)来反映多环芳烃产生的原 因. 较低的 LMW-PAHs/HMW-PAHs 值说明多环芳烃 主要由高温高热的燃烧所产生, 而较高的 LMW-PAHs/ HMW-PAHs 值则表明多环芳烃可能主要来自低温燃 烧或石油燃烧. 珠穆朗玛峰地区土壤中 LMW-PAHs/ HMW-PAHs 值在 3 393 的水平, 植物体中 LMWPAHs/HMW-PAHs值也较高, 为 4 36. 这比Mai等人 ${ }^{[35]}$ 在湖芯中所观察到的数据(0.6 1.4)高了数百倍. 虽 然多环芳烃在随着大气往高海拔地区传输的过程中 低环芳烃的迁移能力强于高环芳烃的迁移能力, 有 可能造成高低环芳烃的组成分异现象, 从而使 LMW-PAHs/HMW-PAHs值偏高, 但是我们仍然不能 排除PAHs排放源中含有大量的由一些低温的燃烧活 动所导致多环芳烃的可能性. 这些燃烧活动可能包 
括家用取暖对煤、植物或者油气的燃烧过程.

事实上每一种燃烧活动都不可避免地既包括高 温燃烧也包括低温燃烧. 诸如汽车尾气、煤/焦炭的燃 烧、森林大火、生物物质燃烧以及工业冶炼都会将多 环芳烃排放到环境当中. 这些燃烧活动都会相应产 生一些具有较高分子量的多环芳烃. 很多研究集中 于对燃烧介质所产生的多环芳烃的组成进行分析和 归纳, 企图找出一些特征指标来反演多环芳烃的排 放源. Colmsjo等 ${ }^{[36]}$ 和Rogge $\mathrm{e}^{[37]}$ 等人对汽车尾气排放 的多环芳烃做了研究. Dominguez ${ }^{[38]}$ 等人则主要对各 种煤燃烧所产生的多环芳烃做了研究. Jenkins ${ }^{[39]}$ 等 人的研究重点是木材燃烧所产生的多环芳烃. 他们 的研究结果得出的共同结论就是: 样品中苯并 [a]蒽 和䓛的比值 $(\mathrm{B}[\mathrm{a}] \mathrm{A} / \mathrm{Chr})$ 和苯并 $[\mathrm{b}]$ 苂蒽与苯并 $[\mathrm{k}]$ 荧蒽 的比值 $(\mathrm{B}[\mathrm{b}] \mathrm{F} / \mathrm{B}[\mathrm{k}] \mathrm{F})$ 可以用来判定产生多环芳烃的 来源. 图 5 列出了各种人为燃烧过程所产生的特征比 值. 如图所示, 汽车尾气所产生的B $[\mathrm{a}] \mathrm{A} / \mathrm{Chr}$ 值约为 $0.53 \pm 0.06$, 而 $\mathrm{B}[\mathrm{b}] \mathrm{F} / \mathrm{B}[\mathrm{k}] \mathrm{F}$ 值约为 $1.26 \pm 0.19$. 煤燃 烧所产生的 $\mathrm{B}[\mathrm{a}] \mathrm{A} / \mathrm{Chr}$ 值约为 $1.11 \pm 0.06$, 而 $\mathrm{B}[\mathrm{b}] \mathrm{F} / \mathrm{B}[\mathrm{k}] \mathrm{F}$ 值约为 $3.70 \pm 0.17$. 从珠穆朗玛峰地区植 被中检测到的 $\mathrm{B}[\mathrm{a}] \mathrm{A} / \mathrm{Chr}$ 和 $\mathrm{B}[\mathrm{b}] \mathrm{F} / \mathrm{B}[\mathrm{k}] \mathrm{F}$ 来看, $\mathrm{B}[\mathrm{a}] \mathrm{A} / \mathrm{Chr}=0.695$ 而 $\mathrm{B}[\mathrm{b}] \mathrm{F} / \mathrm{B}[\mathrm{k}] \mathrm{F}=1.59$, 落入汽车尾 气和煤燃烧的共同区域(图 5), 并且更加接近于汽车 尾气的比值. 我们利用Bidleman和Falconer所提出的 两组分混和模型 ${ }^{[40]}$ 分别计算了汽车尾气和煤燃烧对 产生于高热的多环芳烃的相对贡献. 计算结果表

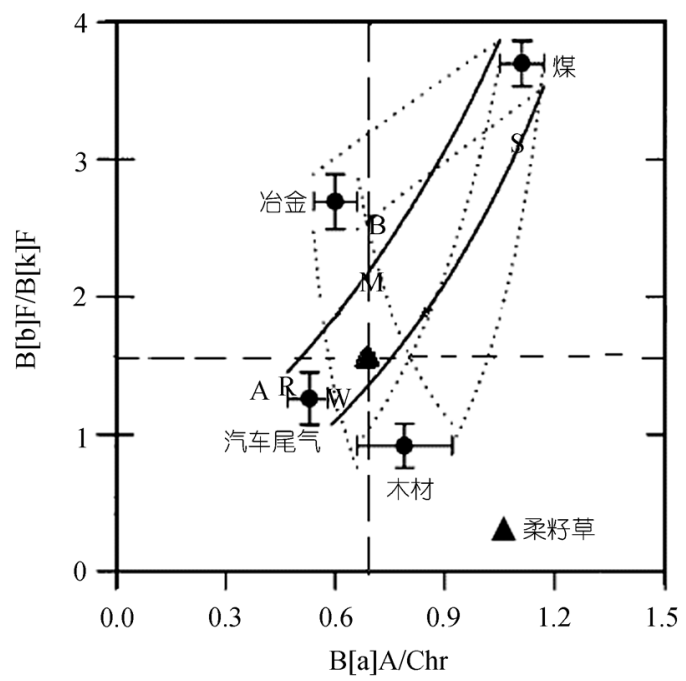

图 5 来源于煤、汽车尾气、木材以及冶金的多环芳烃组 成比值 $\mathrm{B}[\mathrm{a}] \mathrm{A} / \mathrm{Chr}$ 与珠穆朗玛峰柔籽草中多环芳烃组成比

值 $\mathrm{B}[\mathrm{b}] \mathrm{F} / \mathrm{B}[\mathrm{k}] \mathrm{F}$
明珠穆朗玛峰地区高热多环芳烃 $66 \%$ 来源于汽车尾 气的排放, 剩余 $34 \%$ 是源自煤高温燃烧. 珠穆朗玛峰 地区低分子量的多环芳烃主要来源于家用取暖对煤、 植物或者油气的燃烧过程, 而高分子量多环芳烃则 是由汽车尾气和煤的高温燃烧所产生的. 珠穆朗玛 峰地区与印度和中国相毗邻. 印度和中国同时也是 世界上人口最多的国家. 其他邻近地区如巴基斯坦 和孟加拉的人口也在剧烈的增长. 由人口增长所带 来的公路的发展, 机动车使用的增加都会导致汽车 尾气所排放的多环芳烃的增加. 无论是家用取暖、煤 燃烧还是汽车尾气排放的各种多环芳烃都会随着季 风的传输而到达珠穆朗玛峰.

\section{4 多环芳烃的大气传输路径}

珠穆朗玛峰地区污染物的传输路径受到该地区 季节性气候的显著影响. 在夏季的 6 9 月, 珠穆朗玛 峰地区的水汽输送主要来自印度季风. 季风主要来 自印度洋, 经过印度中部和孟加拉湾到达喜马拉雅 中段. 冬季南下的西风带控制了珠穆朗玛峰地区的 气候. 源自大西洋和地中海海水蒸发的水汽途经伊 朗高原、印度北部及巴基斯坦等人类活动频繁的地 区 $^{[41]}$. 污染物的传输会明显地受到气象条件的影响. 典型的气候特点是决定多环芳烃传输的主要因素. 珠穆朗玛峰地区的气候受到明显的冬夏季风影响, 季节性变化特别明显. 因此, 珠穆朗玛峰是研究污染 物传输季节性变化的良好区域 ${ }^{[42]}$. 在冬夏季风各自 的传输途径以及盛行时间方面我们没有做更多的研 究, 因为很多研究者都做了详尽的工作, 对冬夏季风 的起止时间做了很好的界定 ${ }^{[43]}$. 一些学者在研究多 环芳烃的季节性含量变化的时候发现, 冬季由于燃 烧活动的增加, 导致了环境中有高含量的多环芳烃. 而且, 来自印度和巴基斯坦的数据也证明在这些地 区冬季大量的燃烧活动显著增加了大气中多环芳烃 的含量 ${ }^{[44,45]}$. 当西风经过这些多环芳烃排放的地区 就会将这些污染物带到珠穆朗玛峰地区乃至整个青 藏高原. 事实上, 无论是西风还是印度季风都可以将 污染排放源区的多环芳烃带往珠穆朗玛峰. 只不过 冬季西风盛行且多环芳烃排放更加严重, 相比印度 季风而言西风可能将更多的多环芳烃携带到珠穆朗 玛峰地区. 随着西风以及印度季风的盛行, 污染物质 经过长距离的大气搬运就会降落在珠穆朗玛峰地区, 储存于冰雪、土壤之中或者被植物吸附. 季风对污染 物的输送起非常重要的作用. 


\section{5 结论}

珠穆朗玛峰地区土壤及植被中多环芳烃的含量 主要是由于大气远距离输送而来, 而且该地区土壤 中多环芳烃的含量与地球上其他边远地区土壤中的 含量接近, 基本上可以代表北半球远离污染源地区 土壤中多环芳烃的背景值. 土壤中多环芳烃的总量 随着海拔高度的升高有升高的趋势，表明珠穆朗玛 峰高海拔处更易于受到多环芳烃的影响. 珠穆朗玛 峰地区的多环芳烃主要是由一些低温燃烧活动, 诸 如家用取暖所导致的, 而由高热产生的多环芳烃则 主要为汽车尾气的排放物和煤燃烧排放物. 季风对 于多环芳烃的传输起非常重要的作用, 冬季西风盛 行, 又是中亚及印度北部多环芳烃大量排放的季节, 西风可以将这些人类活动频繁地区所排放的多环芳 烃带到青藏高原珠穆朗玛峰地区.

致谢 本工作受国家自然科学基金创新群体项目(批准号: 40121101)、国家自然科学基金项目(批准号：40401054, 40501018)和国家重点基础研究发展计划项目 (批准号: 2005 CB422004)资助.

\section{参考文献}

1 Ottar B. The transfer of airborne pollutants to the arctic region. Atmos Environ, 1981, 15: 1439-1445[DOI]

2 Wania F W, Mackay D. Tracking the distribution of persistent organic pollutants. Environ Sci Technol, 1996, 30: 390A-396A

3 Gouin T, Mackay D, Jones K C, et al. Evidence for the "grasshopper" effect and fractionation during long-range atmospheric transport of organic contaminants. Environ Pollut, 2004, 128: 139-148[DOI]

4 Daly G L, Wania F. Organic contaminants in mountains. Environ Sci Technol, 2005, 39: 385-398[DOI]

5 Gregor D J, Gummer W D. Evidence of atmospheric trans port and deposition of organochlorine pesticides and polychlorinated biphenyls in Arctic snow. Environ Sci Technol, 1989, 23: 15281531

6 Arctic Monitoring and Assessment Programm (AMAP). AMAP assessment 2002: persistent organic pollutants in the Arctic. AMAP Reports. 2002

7 Li Y F, MacDonald R W, Jantunen L M M, et al. The transport of $\beta$-hexachlorocyclohexane to the western Arctic Ocean: a contrast to $\alpha$-HCH. Sci Total Environ, 2002, 291: 229-246[DOI]

8 Bailey R, Barrie L A, Halsall C J, et al. Atmospheric organochlorine pesticides in the western Canadian Arctic: Evidence of transpacific transport. J Geophy Res, 2000,105: 11805-11811[DOI]

9 Hanot L, Domine F. Evolution of the surface area of a snow layer. Environ Sci Technol, 1999, 33: 4250-4255[DOI]

10 Pfirman S L, Eicken H, Bauch D, et al. The potential transport of pollutants by Arctic sea ice. Sci Total Environ, 1995, 159: 129$146[\mathrm{DOI}]$

11 Jaward F M, Farrar N, Harner T, et al. Passive air sampling of PCBs, PBDEs, and organochlorine pesticides across Europe. Environ Sci Technol, 2004, 38: 34-41[DOI]

12 Campbell L. The use of stable isotope ratios to discern organochlorine bioaccumulation patterns in a subalpine Rocky Mountain lake foodweb. Dissertation for the Msc degree. Edmonton: University of Alberta, 1997

13 Braekevelt E. Effects of suspended glacial particles on the bioavailability of hydrophobic organic contaminants in two subalpine lakes in the Canadian Rocky Mountains. Dissertation for the Msc degree. Edmonton: University of Alberta, 2000

14 Peters A J, Gregor D J, Teixeira C F, et al. The recent depositional trend of polycyclic aromatic hydrocarbons and elemental carbon to the Agassiz Ice cap, Ellesmere Island, Canada. Sci Total Environ, 1995,160/161: 167-179

15 Fernandez P, Carrera G, Grimalt J O. Persistent organic pollutants in remote freshwater ecosystems. Aquat Sci, 2005, 67: 263-273

16 Kelly B C, Gobas F A P C. Bioaccumulation of persistent organic pollutants in Lichen-Caribou-Wolf food chains of Canada's central and western Arctic. Environ Sci Technol, 2001, 35: 325-334

17 Loewen M D, Sharma S, Tomy G, et al. Persistent organic pollutants and mercury in the Himalaya. Aquat Eco Heal Mana, 2005, 8: $223-233$

18 Bodnar E, Hlavay J. Atmospheric deposition of polycyclic aromatic hydrocarbons on the Lake Balaton, Hungary. Micro J, 2005, 79: $213-220[\mathrm{DOI}]$

19 Migaszewski Z M, Determining organic compound ratios in soils and vegetation of the Holy Cross Mt. Poland. Water Air and Soil Pollut, 1999, 111: 123-138[DOI]

20 Fang J Y, Kanzaki M, Wang X P, et al. Community structure of alpine sparse vegetataion and effects of microtopography in Pushila, Everest-Choyu region, Tibet. China Biodiversity Sci, 2004, 12: 190-199

21 Jones K C, Stratford J A, Waterhouse K S, et al. Organic contaminants in Welsh soils: Polynuclear aromatic hydrocarbons. Environ Sci Technol, 1989, 23: 540—550[DOI]

22 Maliszewska-Kordybach B. Polycyclic aromatic hydrocarbons in agricultural soils in Poland: preliminary proposals for criteria to evaluate the level of soil contamination. Appl Geochem, 1996, 11: 121-127[DOI]

23 Mazzera D, Hayes T, Lowenthal D, et al. Quantification of polycyclic aromatic hydrocarbons in soil at McMurdo Station, Antarctica. Sci Total Environ, 1999, 229: 65-71[DOI]

24 Aislabie J, Balks M, Astori N, et al. Polycyclic aromatic hydrocarbons in fuel-oil contaminated soils, Antarctica. Chemosphere, 1999, 39: 2201-2207[DOI]

25 Yunker M B, Snowdon L R, Macdonald R W, et al. Polycyclic aromatic hydrocarbon composition and potential sources for sediment samples from the Beaufort and Barents Seas. Environ Sci Technol, 1996, 30: 1310-1320[DOI] 
26 Gschwend P M, Hites R A. Fluxes of polycyclic aromatic hydrocarbons to marine and lacustrine sediments in the northern United States. Geoch Cosmoch Acta, 1981, 45: 2359-2367[DOI]

27 Sicre M A, Marty J C, Saliot A, et al. Aliphatic and aromatic hydrocarbons in different sized aerosols over the Mediterranean Sea: Occurrence and origin. Atmos Environ, 1987, 21: 2247-2259[DOI]

28 Simon R, Colom-Altes M, Grimalt J O, et al. Background levels of atmospheric hydrocarbons, sulphate and nitrate over the western Mediterranean. Atmos Environ, 1991, 25A: 1463-1471 , Jones $\mathrm{K}$ C, Johnston A E. The polynuclear aromatic hydrocarbon (Pah) content of herbage from a long-term grassland experiment. Atmos Environ A-General Topics, 1992, 26: 1299$1307[\mathrm{DOI}]$

30 Miguel A H. Seasonal variation of the particle size distribution of polycyclic aromatic hydrocarbons and of major aerosol species in Claremont, California. Atmos Environ, 2004, 38: 3241-3251[DOI]

31 Liu X, Zhang G, Jones K C, et al. Compositional fractionation of polycyclic aromatic hydrocarbons (PAHs) in mosses (Hypnum plumaeformae WILS.) from the northern slope of Nanling Mountains, South China. Atmos Environ, 2005, 39: 5490-5499[DOI]

32 Blais J M, Schindler D W, Muir D C G, et al. Accumulation of persistent organochlorines in mountains of western Canada. Nature, 1998, 395: 585-588[DOI]

33 Blais J M, Wilhelm F, Kidd K A, et al. Concentrations of organochlorine pesticides and PCBs in amphipods (Gammarus lacustris ) along an elevation gradient in mountain lakes of western Canada. Environ Toxico Chem, 2003, 22: 2605-2613[DOI]

34 Coulon F, Pelletier E, Gourhant L, et al. Effects of nutrient and temperature on degradation of petroleum hydrocarbons in contaminated sub-Antarctic soil. Chemosphere, 2005, 58: 1439-1448[DOI]

35 Mai B X, Qi S H, Zeng E Y, et al. Distribution of polycyclic aromatic hydrocarbons in the coastal region off Macao, China: Assessment of input sources and transport pathways using compositional analysis. Environ Sci Technol, 2003, 37: 4855-4863[DOI]
36 Colmsjo A L, Zebuhr Y U, Ostman C E. Polynuclear aromatic compounds in the ambient air of Stockholm. Chemosphere,1986, 15: $169-182[\mathrm{DOI}]$

37 Rogge W F, Hildemann L M, Mazurek M A, et al. Sources of fine organic aerosol. 2. Noncatalyst and catalyst-equipped automobiles and heavy-duty diesel trucks. Environ Sci Technol, 1993, 27: 636 - 651[DOI]

38 Dominguez A, Alvarez R, Blanco C G, et al. Chromatographic evaluation of some selected polycyclic aromatic hydrocarbons of coal tars produced under different coking conditions and pitches derived from them. J Chromato, 1996, 719: 181-194[DOI]

39 Jenkins B M, Jones A D, Turn S Q, et al. Particle concentrations, gas-particle partitioning, and species intercorrelations for polycyclic aromatic hydrocarbons (PAHs) emitted during biomass burning. Atmos Environ, 1996, 30: 3825-3835[DOI]

40 Bidleman T F, Falconer R L. Enantiomer ratios for apportioning two sources of chiral compounds. Environ Sci Technol, 1999, 33: $2299-2301[\mathrm{DOI}]$

41 田立德, 姚檀栋, White J W C, 等. 喜马拉雅山中段过量氝与西 风带水气输送有关. 科学通报, 2005, 50(7): 1026-1030

42 Kang S, Mayewski P A, Qin D, et al. Seasonal differences in snow chemistry from the vicinity of Mt. Everest, central Himalayas. Atmos Environ, 2004, 38: 2819-2829[DOI]

43 Lelieveld J, Crutzen P J, Ramanathan V. The India ocean experiment: widespread air pollution from south and southeast Asia. Science, 2001, 291: 1031-1036[DOI]

44 Smith D J T, Harrison R M, Luhana L, et al. Concentrations of particulate airborne polycyclic aromatic hydrocarbons and metals collected in Lahore, Pakistan. Atmos Environ, 1996, 30: 40314040[DOI]

45 Bhargava A, Khanna R N, Bhargava S K, et al. Exposure risk to carcinogenic PAHs in indoor-air during biomass combustion whilst cooking in rural India. Atmos Environ, 2004, 38: 4761 -4767[DOI]

(2006-05-22 收稿, 2006-09-30 接受) 